\title{
Behavioral changes following HIV seroconversion during the historical expansion of HIV treatment in the United States
}

\section{Supplemental Materials}

\author{
Appendix Tables A1-A5
}

2 ..... Table A1. Longitudinal associations of HIV seroconversion with subsequent risk behaviors with mediation by physical and mental health variables among MACS participants who seroconverted during observation $(\mathrm{n}=558)$

5 ..... Table A2. Heterogeneous associations of HIV seroconversion with subsequent risk behaviors by exposure to HIV treatment with mediation by physical health variables among MACS participants who seroconverted during observation $(\mathrm{n}=558)$

9 廆Table A3. Heterogeneous associations of HIV seroconversion with subsequent risk behaviors by exposure to HIV treatment with mediation by mental health variable among MACS participants who seroconverted during observation $(n=558)$

13 廆 Table A4. One-period attrition with and without deaths included

16 䖉T Table A5. Associations of HIV seroconversion with subsequent risk behaviors: full sample pooled cross section $(\mathrm{n}=4616)$ 


\section{Appendix Tables}

hal associations of HIV seroconversion with subsequent risk behaviors with mediation by physical and mental health variables among MACS participants who seroconverted during

Dependent Variable: dummy variable ?in $t+1$

\begin{tabular}{ccccc}
\hline & insertive anal sex with & receptive anal sex with & & \\
sex with & $2+$ partners & $2+$ partners & 3+ drinks/day & smoke $1 / 2+$ \\
$2+$ partners & $\mid 2+$ partners & $\mid 2+$ partners & if drinking & packs/day monthly+ \\
\hline
\end{tabular}

Panel A: Mediation by Physical Health

(1)

(2)

(3)

(4)

(5)

(6)

re-conversion)

$\begin{array}{ccc}0 ? 443 & 0 ? 495 & 1 ? 451 \\ (0 \text { ?305, 0?645) } & (0 ? 288,0 ? 849) & (0 ? 871,2 ? 418 \\ \text { Yes } & \text { Yes } & \text { Yes } \\ \text { Yes } & \text { Yes } & \text { Yes } \\ \text { Yes } & \text { Yes } & \text { Yes } \\ \text { Yes } & \text { Yes } & \text { Yes }\end{array}$

?451

$0 ? 883$

(0?627, 1?243)

(0?717, 2?380)

$1 ? 307$

$0 ? 776$
Yes
Yes

Yes

Yes

Yes
Yes

Yes

Yes

Yes
Yes

Yes

Yes

Yes 


\begin{tabular}{|c|c|c|c|c|c|c|}
\hline \multirow{3}{*}{ ervations } & 8,447 & 3,641 & 3,603 & 6,612 & 3,270 & 5,245 \\
\hline & $0 ? 566$ & $0 ? 431$ & $0 ? 462$ & $0 ? 412$ & $0 ? 478$ & $0 ? 392$ \\
\hline & $0 ? 112$ & $0 ? 073$ & $0 ? 049$ & $0 ? 057$ & $0 ? 126$ & $0 ? 123$ \\
\hline & \multicolumn{6}{|c|}{ Panel B: Mediation by Mental Health } \\
\hline & (8) & (9) & $(10)$ & $(11)$ & $(12)$ & (13) \\
\hline re-conversion) & $0 ? 351$ & $0 ? 369$ & $1 ? 045$ & $0 ? 659$ & $1 ? 208$ & $0 ? 748$ \\
\hline & $(0 ? 248,0 ? 498)$ & $(0 ? 224,0 ? 607)$ & $(0 ? 642,1 ? 703)$ & $(0 ? 473,0 ? 919)$ & $(0 ? 698,2 ? 088)$ & $(0 ? 466,1 ? 202$ \\
\hline ale Control & Yes & Yes & Yes & Yes & Yes & Yes \\
\hline ts & Yes & Yes & Yes & Yes & Yes & Yes \\
\hline ffects & Yes & Yes & Yes & Yes & Yes & Yes \\
\hline rols & Yes & Yes & Yes & Yes & Yes & Yes \\
\hline rvations & 8,672 & 3,756 & 3,654 & 6,749 & 3,438 & 5,331 \\
\hline
\end{tabular}


$0 ? 572$

0?103
$0 ? 439$

$0 ? 068$
0?459

0?047
$0 ? 418$

0?059
$0 ? 480$

0?127
$0 ? 403$

0?126

rts odds ratios from logit regressions showing the associations of HIV seroconversion with subsequent risk behaviors, with mediation by physical health (columns (1)-(7)) and ment are restricted to participants who seroconverted $(n=558)$. Each column is a separate regression on one of the seven outcomes of interest (defined in the text) representing sexual bel onal drug use. Columns (1)-(7) control for CD4 count and CD4 count squared. Columns (8)-(14) control for CES-D score (out of 60). All regressions also control for (results not sh ixed effects, time trend and its interaction with high school degree, age interacted with high school degree, and time trend interacted with baseline level of each corresponding depe ns is less than the full sample and changes across columns because of missing values in the dependent variables, which differ across columns. Specifically, the variables in columns 8-49, and are conditional on someone having 2+ male sexual partners; the variable in column (4) and (11) is conditional on having any drink in the period; we miss additional obser s a consequence of (1) missing self-reported data, (2) requiring one-period forward values, and (3) logit estimation dropping observations because of all positive/negative outcomes ses) are based on standard errors adjusted for clustering at the individual level. 
Table A2. Heterogeneous associations of HIV seroconversion with subsequent risk behaviors by exposure to HIV treatment with mediation by physical health variables among MACS participants who seroconverted during observation ( $\mathrm{n}=558)$

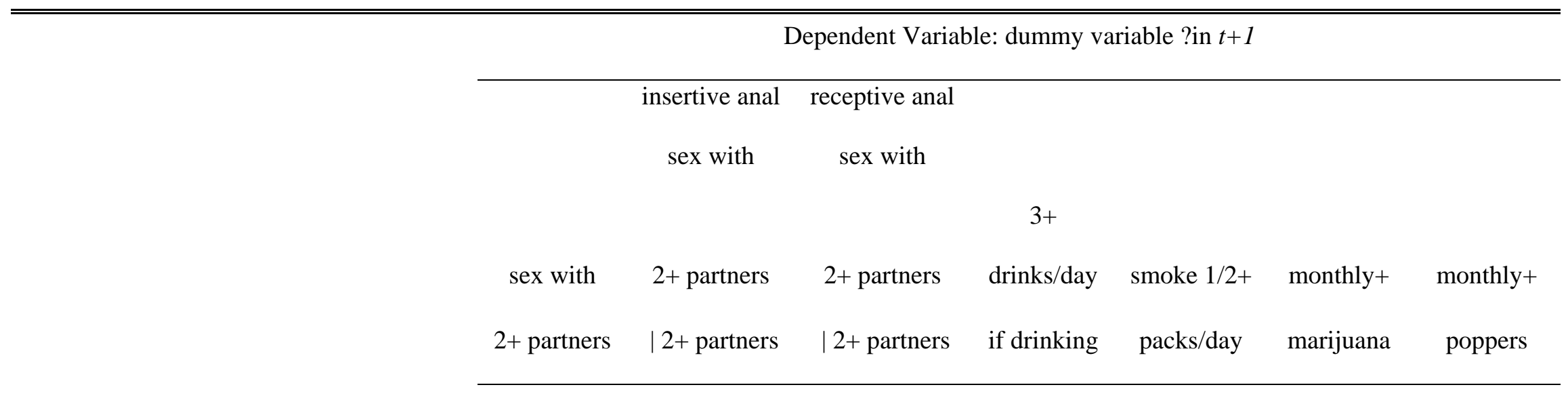

Panel A: Definition of exposure to HIV treatment: post-1996

(1)

$(2)$

\begin{tabular}{|c|c|c|c|c|c|c|c|}
\hline \multirow[t]{3}{*}{ conversion } & $0 ? 468$ & $0 ? 579$ & $1 ? 300$ & 0?902 & $1 ? 571$ & $0 ? 708$ & 0?935 \\
\hline & (0?317, & (0?323, & (0?754, & (0?636, & (0?820, & (0?432, & (0?600, \\
\hline & 0?690) & 1?041) & 2?241) & 1?281) & 3?011) & 1?161) & 1?457) \\
\hline post-1996: post-conversion vs. pre- & $0 ? 286$ & $0 ? 277$ & $2 ? 204$ & $0 ? 768$ & $0 ? 507$ & $1 ? 615$ & $0 ? 424$ \\
\hline
\end{tabular}

pre-1996: post-conversion vs. pre-

conversion

post-1996: post-conversion vs. pre-
(3)

(4)

(5)

(6)

(7) 
conversion

$\begin{array}{lllllll}(0 ? 120, & (0 ? 145, & (1 ? 012, & (0 ? 312, & (0 ? 214, & (0 ? 513, & (0 ? 177, \\ 0 ? 682) & 0 ? 527) & 4 ? 801) & 1 ? 887) & 1 ? 201) & 5 ? 085) & 1 ? 015)\end{array}$

Quadratic CD4 Control

Individual Fixed Effects

Survey Wave Fixed Effects

Individual-Level Controls

Individual-Wave Observations

Pre-1996=Post-1996 (P-value)

Dep. Var. Mean

Adjusted R-squared

$\begin{array}{cc}\text { Yes } & \text { Yes } \\ \text { Yes } & \text { Yes } \\ \text { Yes } & \text { Yes } \\ \text { Yes } & \text { Yes }\end{array}$

Yes

8,447

$0 ? 266$

$0 ? 566$

$0 ? 113$

$0 ? 431$

$0 ? 075$

Yes
Yes
Yes
Yes

Yes

Yes

Yes

Yes

3,603

$0 ? 176$

$0 ? 462$

3,641
$0 ? 028$
$0 ? 431$
$0 ? 075$

$0 ? 051$

6,612

3,270

5,245

6,152

$0 ? 730 \quad 0 ? 018$

$0 ? 169$

0?059

$0 ? 412 \quad 0 ? 478$

0 ?392

$0 ? 348$

$0 ? 058$

$0 ? 129$

$0 ? 124$

$0 ? 130$

Panel B: Definition of exposure to HIV treatment: HIV treatment initiation
(8)
(9)
(10)
(11)
(12)
(13)
(14) 
without treatment: post-conversion vs.

pre-conversion

$\begin{array}{ccccccc}0 ? 451 & 0 ? 582 & 1 ? 476 & 0 ? 911 & 1 ? 342 & 0 ? 773 & 0 ? 877 \\ (0 ? 309, & (0 ? 342, & (0 ? 881, & (0 ? 650, & (0 ? 744, & (0 ? 481, & (0 ? 566, \\ 0 ? 660) & 0 ? 991) & 2 ? 473) & 1 ? 278) & 2 ? 422) & 1 ? 241) & 1 ? 357)\end{array}$

with treatment: post-conversion vs. pre-

conversion

$\begin{array}{ccccccc}0 ? 335 & 0 ? 293 & 1 ? 379 & 0 ? 552 & 0 ? 716 & 0 ? 814 & 0 ? 523 \\ (0 ? 213, & (0 ? 149, & (0 ? 757, & (0 ? 343, & (0 ? 311, & (0 ? 417, & (0 ? 281, \\ 0 ? 526) & 0 ? 575) & 2 ? 512) & 0 ? 887) & 1 ? 650) & 1 ? 590) & 0 ? 973)\end{array}$

Quadratic CD4 Control

Yes

Yes

Yes

Yes

Yes

Yes

Individual Fixed Effects

Yes

Yes

Yes

Yes

Yes

Yes

Survey Wave Fixed Effects

Individual-Level Controls

Yes

Yes

Yes

Yes

Yes

Yes

Yes

Yes

Yes

Yes

Yes

Yes

Individual-Wave Observations

8,447

3,641

3,603

6,612

3,270

5,245

6,152

Pre-treatment=Post-treatment (P-value)

0?070 $\quad 0 ? 002$

$0 ? 738$

$0 ? 004$

$0 ? 096$

$0 ? 825$

$0 ? 013$

Dep. Var. Mean

$0 ? 566$

$0 ? 431$

$0 ? 462$

$0 ? 412$

$0 ? 478$

$0 ? 392$

$0 ? 348$

Adjusted R-squared

0?113 $0 ? 079$

0?049

0?060

0?130

0 ?123

$0 ? 132$ 
Notes: The table reports odds ratios from logit regressions showing the heterogeneous associations of HIV seroconversion with subsequent risk behaviors by exposure to HIV treatment, with mediation by physical health variables (CD4 count and CD4 count squared). Regressions are restricted to participants who seroconverted $(n=558)$. Each column is a separate regression on one of the seven outcomes of interest (defined in the text) representing sexual behavior, drinking, smoking, and recreational drug use. All regressions also control for (results not shown) individual fixed effects, survey wave fixed effects, indicator of exposure to HIV treatment, time trend and its interaction with high school degree, age interacted with high school degree, and time trend interacted with baseline level of each corresponding dependent variable. The number of observations is less than the full sample and changes across columns because of missing values in the dependent variables, which differ across columns. Specifically, the variables in columns (2), (3), (9) and (10) are covered in waves 8-49, and are conditional on someone having 2+ male sexual partners; the variable in column (4) and (11) is conditional on having any drink in the period; we miss additional observations for all the dependent variables as a consequence of (1) missing self-reported data, (2) requiring one-period forward values, and (3) logit estimation dropping observations because of all positive/negative outcomes. All 95\% Confidence Intervals (in parentheses) are based on standard errors adjusted for clustering at the individual level. 
Table A3. Heterogeneous associations of HIV seroconversion with subsequent risk behaviors by exposure to HIV treatment with mediation by mental health variable among MACS participants who seroconverted during observation $(n=558)$

Dependent Variable: dummy variable ?in $t+1$

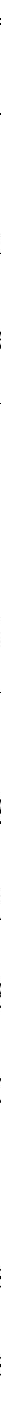




\begin{tabular}{|c|c|c|c|c|c|c|}
\hline Wave Fixed Effects & Yes & Yes & Yes & Yes & Yes & Yes \\
\hline dual-Level Controls & Yes & Yes & Yes & Yes & Yes & Yes \\
\hline dual-Wave Observations & 8,672 & 3,756 & 3,654 & 6,749 & 3,438 & 5,331 \\
\hline 96=Post-1996 (P-value) & $0 ? 105$ & $0 ? 090$ & 0?390 & $0 ? 886$ & $0 ? 005$ & 0?330 \\
\hline ar. Mean & $0 ? 572$ & $0 ? 439$ & $0 ? 459$ & $0 ? 418$ & $0 ? 480$ & $0 ? 403$ \\
\hline ed R-squared & $0 ? 105$ & $0 ? 069$ & $0 ? 048$ & $0 ? 059$ & $0 ? 132$ & $0 ? 127$ \\
\hline
\end{tabular}

Panel B: Definition of exposure to HIV treatment: HIV treatment initiation

\begin{tabular}{|c|c|c|c|c|c|}
\hline (8) & (9) & (10) & (11) & (12) & (13) \\
\hline $0 ? 368$ & $0 ? 450$ & $1 ? 110$ & $0 ? 706$ & $1 ? 327$ & $0 ? 742$ \\
\hline 57, 0?527) & $(0 ? 276,0 ? 735)$ & $(0 ? 680,1 ? 810)$ & (0?509, 0?979) & $(0 ? 760,2 ? 316)$ & $(0 ? 465,1 ? 185)$ \\
\hline $0 ? 235$ & $0 ? 226$ & $0 ? 903$ & $0 ? 416$ & $0 ? 660$ & $0 ? 785$ \\
\hline $53,0 ? 360)$ & $(0 ? 121,0 ? 422)$ & $(0 ? 502,1 ? 626)$ & $(0 ? 256,0 ? 676)$ & $(0 ? 305,1 ? 430)$ & $(0 ? 399,1 ? 542)$ \\
\hline
\end{tabular}


dual Fixed Effects

y Wave Fixed Effects

dual-Level Controls

dual-Wave Observations

eatment=Post-treatment (P-value)

Var. Mean

ted R-squared
Yes

Yes

Yes

$0 ? 005$

$0 ? 572$

0?106
Yes

Yes

Yes

3,756

0?001

0?439

0?074
Yes

Yes

Yes

3,654

0 ?313

$0 ? 459$

0?047
Yes

Yes

Yes

6,749

$0 ? 004$

$0 ? 418$

0?062
Yes

Yes

Yes

3,438

$0 ? 062$

$0 ? 480$

$0 ? 133$
Yes

Yes

Yes

5,331

0?821

0?403

0?126
Yes$$
\text { Yes }
$$

Ye
6,39
$0 ? 00$

$0 ? 35$

$0 ? 11$

The table reports odds ratios from logit regressions showing the heterogeneous associations of HIV seroconversion with subsequent risk behaviors by exposure to HIV treatment, tion by mental health variable (CES-D score out of 60). Regressions are restricted to participants who seroconverted ( $\mathrm{n}=558$ ). Each column is a separate regression on one of the se nes of interest (defined in the text) representing sexual behavior, drinking, smoking, and recreational drug use. All regressions also control for (results not shown) individual fixed , survey wave fixed effects, indicator of exposure to HIV treatment, time trend and its interaction with high school degree, age interacted with high school degree, and time trend ted with baseline level of each corresponding dependent variable. The number of observations is less than the full sample and changes across columns because of missing values in dent variables, which differ across columns. Specifically, the variables in columns (2), (3), (9) and (10) are covered in waves 8-49, and are conditional on someone having 2+ male rs; the variable in column (4) and (11) is conditional on having any drink in the period; we miss additional observations for all the dependent variables as a consequence of (1) miss ported data, (2) requiring one-period forward values, and (3) logit estimation dropping observations because of all positive/negative outcomes. All 95\% Confidence Intervals (in heses) are based on standard errors adjusted for clustering at the individual level. 
Table A4. One-period attrition with and without deaths included

\begin{tabular}{|c|c|c|c|c|c|c|}
\hline & \multicolumn{6}{|c|}{ Probability of dropping out of sample in $t+1 \mid$ in sample in $t$} \\
\hline & \multicolumn{3}{|c|}{$\underline{\text { Sample with Deaths }}$} & \multicolumn{3}{|c|}{$\underline{\text { Sample without Deaths }}$} \\
\hline & (1) & $(2)$ & (3) & $(4)$ & (5) & (6) \\
\hline \multirow[t]{2}{*}{ Sex with $2+$ partners } & $-0 ? 007$ & $-0 ? 002$ & & $0 ? 006$ & $0 ? 005$ & \\
\hline & $(0 ? 016)$ & $(0 ? 018)$ & & $(0 ? 019)$ & $(0 ? 022)$ & \\
\hline \multirow[t]{2}{*}{ Smoke $1 / 2+$ packs/day } & $0 ? 007$ & $0 ? 013$ & $0 ? 000$ & $0 ? 002$ & $0 ? 005$ & $0 ? 012$ \\
\hline & $(0 ? 019)$ & $(0 ? 020)$ & $(0 ? 033)$ & $(0 ? 023)$ & $(0 ? 024)$ & $(0 ? 038)$ \\
\hline \multirow[t]{2}{*}{ Monthly+ marijuana } & $0 ? 002$ & $0 ? 004$ & $-0 ? 011$ & $-0 ? 030$ & $-0 ? 026$ & $-0 ? 043$ \\
\hline & $(0 ? 017)$ & $(0 ? 018)$ & $(0 ? 031)$ & $(0 ? 018)$ & $(0 ? 020)$ & $(0 ? 034)$ \\
\hline \multirow[t]{2}{*}{ Monthly+ poppers } & $0 ? 010$ & $-0 ? 002$ & $0 ? 007$ & $0 ? 030$ & $0 ? 015$ & $0 ? 019$ \\
\hline & $(0 ? 015)$ & $(0 ? 016)$ & $(0 ? 025)$ & $(0 ? 018)$ & $(0 ? 019)$ & $(0 ? 027)$ \\
\hline \multirow[t]{2}{*}{ 3+ drinks/day if drinking } & & $-0 ? 010$ & $-0 ? 007$ & & $-0 ? 015$ & $-0 ? 001$ \\
\hline & & $(0 ? 014)$ & $(0 ? 023)$ & & $(0 ? 016)$ & $(0 ? 024)$ \\
\hline Insertive anal sex with $2+$ partners $\mid 2+$ partners & & & $-0 ? 010$ & & & 0?006 \\
\hline
\end{tabular}




\begin{tabular}{|c|c|c|c|c|c|c|}
\hline & & & $(0 ? 023)$ & & & $(0 ? 026)$ \\
\hline Receptive anal sex with $2+$ partners $\mid 2+$ partners & & & $0 ? 053$ & & & $0 ? 046$ \\
\hline & & & $(0 ? 026)$ & & & $(0 ? 029)$ \\
\hline Post-conversion X sex with $2+$ partners & $-0 ? 019$ & $-0 ? 017$ & $-0 ? 003$ & $-0 ? 016$ & $-0 ? 017$ & \\
\hline & $(0 ? 019)$ & $(0 ? 021)$ & $(0 ? 032)$ & (0?022) & $(0 ? 025)$ & \\
\hline Post-conversion X smoke 1/2+ packs/day & $0 ? 004$ & $0 ? 002$ & $-0 ? 020$ & $0 ? 003$ & $0 ? 008$ & $0 ? 004$ \\
\hline & $(0 ? 021)$ & $(0 ? 021)$ & $(0 ? 039)$ & $(0 ? 027)$ & $(0 ? 028)$ & $(0 ? 047)$ \\
\hline Post-conversion X monthly+ marijuana & $0 ? 022$ & $0 ? 015$ & $0 ? 016$ & $0 ? 054$ & $0 ? 048$ & $0 ? 037$ \\
\hline & $(0 ? 019)$ & $(0 ? 020)$ & (0?036) & (0?023) & $(0 ? 024)$ & $(0 ? 042)$ \\
\hline Post-conversion X monthly+ poppers & $-0 ? 026$ & $-0 ? 011$ & $0 ? 007$ & $-0 ? 038$ & $-0 ? 023$ & $-0 ? 007$ \\
\hline & $(0 ? 018)$ & $(0 ? 019)$ & $(0 ? 029)$ & $(0 ? 023)$ & $(0 ? 024)$ & $(0 ? 033)$ \\
\hline Post-conversion X 3+ drinks/day if drinking & & $0 ? 012$ & $0 ? 026$ & & $0 ? 014$ & $0 ? 007$ \\
\hline & & $(0 ? 017)$ & $(0 ? 027)$ & & $(0 ? 020)$ & $(0 ? 030)$ \\
\hline Post-conversion $\mathrm{X}$ insertive anal sex with $2+$ partners $\mid 2+$ partners & & & $0 ? 012$ & & & $0 ? 004$ \\
\hline & & & $(0 ? 027)$ & & & $(0 ? 031)$ \\
\hline Post-conversion $\mathrm{X}$ receptive anal sex with $2+$ partners $\mid 2+$ partners & & & $-0 ? 070$ & & & $-0 ? 062$ \\
\hline & & & $(0 ? 027)$ & & & $(0 ? 030)$ \\
\hline
\end{tabular}




\author{
Post-1996 Interaction \\ Individual Fixed Effects \\ Survey Wave Fixed Effects \\ Individual-Level Controls
}

Observations

Dep. Var. Mean

Adjusted R-squared

$\begin{array}{cccccc}\text { Yes } & \text { Yes } & \text { Yes } & \text { Yes } & \text { Yes } & \text { Yes } \\ \text { Yes } & \text { Yes } & \text { Yes } & \text { Yes } & \text { Yes } & \text { Yes } \\ \text { Yes } & \text { Yes } & \text { Yes } & \text { Yes } & \text { Yes } & \text { Yes } \\ \text { Yes } & \text { Yes } & \text { Yes } & \text { Yes } & \text { Yes } & \text { Yes }\end{array}$

12,307

10,256

4,597

8,386

7,053

3,567

$0 ? 120$

0?119

$0 ? 108$

0?105

0?104

$0 ? 098$

$0 ? 025$

$0 ? 025$

$0 ? 007$

$0 ? 018$

0?020

$0 ? 008$

Notes: The table reports coefficient estimates from linear probability models showing next-period attrition as a function of current-period risk behaviors using the sample of 558 seroconverters. Estimates shown indicate how attrition is related to outcomes of interest overall and by HIV seroconversion status. Columns (1)-(3) use all MACS participants who seroconverted during observation (n=558). Columns (4)-(6) exclude individuals with known death records (n=290). Columns (1) and (4) use the full sample; columns (2) and (5) are based on a smaller sample where the drinking intensity variable is defined; columns (3) and (6) are based on a smaller sample where insertive and receptive anal sex variables are defined (the sex with 2+ partners variable is omitted due to collinearity). We miss additional observations for all the columns as a consequence of requiring one-period forward values. All regressions also control for (results not shown) individual fixed effects, survey wave fixed effects, time trend and its interaction with high school degree, age interacted with high school degree, and time trend interacted with baseline level of each of 
the included dependent variables. 
Table A5. Associations of HIV seroconversion with subsequent risk behaviors: full sample pooled cross section ( $\mathrm{n}=4616)$

\begin{tabular}{|c|c|c|c|c|c|c|c|}
\hline & \multicolumn{7}{|c|}{ Dependent Variable: dummy variable ?in $t+1$} \\
\hline & & insertive anal & receptive anal & & & & \\
\hline & & sex with & sex with & & & & \\
\hline & sex with & $2+$ partners & $2+$ partners & 3+ drinks/day & smoke $1 / 2+$ & monthly+ & monthly+ \\
\hline & $2+$ partners & | 2+ partners & | 2+ partners & if drinking & packs/day & marijuana & poppers \\
\hline & $(1)$ & $(2)$ & (3) & (4) & (5) & (6) & $(7)$ \\
\hline Currently HIV+ (vs. HIV-) & $0 ? 23$ & $0 ? 47$ & $1 ? 25$ & $0 ? 71$ & $1 ? 59$ & $1 ? 02$ & $0 ? 98$ \\
\hline & $(0 ? 47,0 ? 10)$ & $(0 ? 17,1 ? 00)$ & $(1 ? 48,2 ? 56)$ & $(0 ? 55,1 ? 06)$ & $(0 ? 57,1 ? 02)$ & $(1 ? 74,1 ? 75)$ & $(0 ? 46,1 ? 77)$ \\
\hline Survey Wave Fixed Effects & Yes & Yes & Yes & Yes & Yes & Yes & Yes \\
\hline Individual-Level Controls & Yes & Yes & Yes & Yes & Yes & Yes & Yes \\
\hline Individual-Wave & 55,860 & 23,482 & 23,474 & 46,876 & 56,591 & 56,158 & 56,143 \\
\hline
\end{tabular}


Dep. Var. Mean

Adjusted R-squared
0 ?76

0 ?38
$0 ? 95$

0 ? 16
$0 ? 55$

0 ?33
0?64

0 ?36
0?07

$0 ? 49$
0?65

0?37
$0 ? 14$

$0 ? 44$

Notes: The table reports odds ratios from logit regressions showing the associations of HIV seroconversion with subsequent risk behaviors. Regressions use full sample including always HIV positive, always HIV negative, and seroconverters who became newly infected with HIV during the survey. Each column is a separate regression on one of the seven outcomes of interest (defined in the text) representing sexual behavior, drinking, smoking, and recreational drug use. All regressions also control for (results not shown) survey wave fixed effects, time trend, indicator of recruitment wave, indicator of non-Hispanic White, age, quadratic age, indicator of high school degree, indicator of college degree, and age interacted with high school degree. The number of observations is less than the full sample and changes across columns because of missing values in the dependent variables, which differ across columns. Specifically, the variables in columns (2) and (3) are covered in waves 8-49, and are conditional on someone having 2+ male sexual partners; the variable in column (4) is conditional on having any drink in the period; we miss additional observations for all the dependent variables as a consequence of (1) missing self-reported data, (2) requiring one-period forward values, and (3) logit estimation dropping observations because of all positive/negative outcomes. All 95\% Confidence Intervals (in parentheses) are based on standard errors adjusted for clustering at the individual level. 